\title{
Avaliação da Sustentabilidade da Floresta Nacional de Irati por Meio de Lógica Fuzzy
}

\author{
Deise Regina Lazzarotto ${ }^{1}$
}

${ }^{1}$ Departamento de Engenharia Florestal, Universidade Estadual do Centro Oeste - UNICENTRO, Irati/PR, Brasil

\begin{abstract}
RESUMO
Esta pesquisa avaliou a sustentabilidade de florestas por meio de indicadores processados em Lógica Fuzzy. Utilizou os dados extraídos dos inventários florestais realizados na FLONA de Irati, PR. Com a construção e combinação dos indicadores: INA - Incremento do Número de Árvores; CFL - Crescimento Florestal; TCF - Tendência de Crescimento Florestal foi possível obter o indicador referente à Sustentabilidade da Floresta (SFL) em estudo. Os principais resultados demonstraram que os indicadores INA, CFL e TCF acusaram, respectivamente, um aumento baixo no número de árvores, um crescimento médio para a floresta e também um crescimento médio para a tendência de crescimento da florestal. Por fim, o fragmento da floresta analisado resultou, quanto à sua sustentabilidade, um "estado de equilíbrio". Os objetivos do trabalho foram alcançados e os resultados demonstraram coerência com a situação real.
\end{abstract}

Palavras-chave: unidades de conservação, indicadores florestais, tomada de decisão.

\section{Assessing the Sustainability of Irati National Forest Through Fuzzy Logic}

\begin{abstract}
This research has evaluated the sustainability of Forests by means of indicators processed in Fuzzy Logic. We used data extracted from forest inventories performed at FLONA in Irati-PR. By the construction and combination of indicators such as: INA - Increase the Number of Trees; CFL - Forest Growth; TCF - Forest Growth Trend we obtained the indicator relative to Sustainable Forest (SFL) under study. The main results showed that the indicators INA, CFL and TCF revealed, respectively, a low increase in the number of the trees, an average growth of the forest, as well as an average growth to forest growth trend. Finally, the forest fragment analyzed resulted in a 'balanced state, when regarding sustainability. The objectives of this research were achieved and the results demonstrated consistency with the real situation.
\end{abstract}

Keywords: units of conservation, forest indicators, decision making. 


\section{INTRODUÇÃO}

A preocupação com a preservação e sustentabilidade das florestas, mais especificamente o manejo florestal sustentável, ganha popularidade e aceitação mundial se fazendo presente em muitos debates e discussões públicas que tratam do desenvolvimento de recursos naturais e gestão ambiental (Mendoza \& Prabhu, 2003).

Em 1980, a União Internacional para a Conservação da Natureza - UICN, ao propor a Estratégia Mundial de Conservação - EMC, enfatizou que a conservação e o crescimento não seriam incompatíveis, senão mutuamente dependentes, pois crescer requer recursos naturais, e para cuidar desses são necessários meios econômicos. A UICN, ao chamar essa confluência de "desenvolvimento sustentável", lançou o termo no âmbito internacional já na primeira metade dos anos 1980 e conseguiu que a maior parte dos governos se familiarizasse com esse enfoque (Chang, 2004).

Desde então têm sido crescentes as atividades nas esferas sociais e científicas com o intuito de estimular ações focadas na reposição dos eventuais recursos naturais utilizados. A literatura é rica em publicações sobre a gestão sustentável de uma variedade de recursos naturais, tais como Manejo Florestal, Análise de Ecossistemas, a Produção Agrícola, Sistemas Agroflorestais, Gestão Territorial, Produção Animal, Produção de Alimentos, entre outros (Mendoza \& Prabhu, 2003).

Enquanto se concorda em ter a sustentabilidade como um objetivo de gerenciamento florestal, na prática, meios para avaliá-la ainda não são claros. As dificuldades no desenvolvimento e uso de critérios e indicadores para avaliar a sustentabilidade revela a alta complexidade dos ecossistemas florestais que, portanto, não são totalmente compreendidos (Mendoza \& Prabhu, 2003).

A eficácia das metodologias através da Lógica Fuzzy também pode ser constatada nos estudos realizados pela FEAGRI \& IMECC da UNICAMP, na qual Amendola et al. (2005) afirmam que, a partir do momento em que aprofundaram o contato com alguns processos físicos estudados junto aos especialista da área de Construções Rurais e Ambiência, verificaram a ineficiência do uso de metodologias associadas às ferramentas matemáticas tradicionais nos processos que podem ser modelados matematicamente. Isso os fez procurar novas ferramentas matemáticas o que os levou a concluir pela Teoria de Conjuntos Fuzzy. Suas expectativas foram além das inicialmente propostas.

Amendola et al. (2005) afirmaram que o que se encontra por trás de toda a versatilidade da teoria fuzzy é a possibilidade de modelar e manipular matematicamente informações vagas e imprecisas, naturais da linguagem humana, e, portanto, as fornecidas pelos especialistas ao caracterizar os processos estudados. Esta manipulação é feita a partir de variáveis escolhidas para modelar matematicamente o processo em questão, quando a implicação das variáveis independentes nas dependentes é estabelecida por um conjunto de regras linguísticas baseadas no conhecimento de especialistas.

Dessa forma, os processos operados pela lógica fuzzy têm alcançado as mais diversas áreas de aplicação, principalmente na automatização de processos que variam desde tarefas domésticas até o controle de sofisticados processos industriais (Amendola et al., 2005).

$\mathrm{Na}$ área florestal, a aplicação da lógica fuzzy é mais recente, porém já podemos encontrar diversos estudos e experimentos que buscam justamente a modelagem de fenômenos naturais e fatores de previsibilidade para auxiliar nas tomadas de decisão, destacando-se os temas referentes a incêndios florestais. Como artigos internacionais, citamos os de Akdemir \& Tiryaki (2013) e Chen et al. (2014) e, como artigos nacionais, os de Silva \& Pontes (2011) e Bolfe et al. (2001). Ainda para estudo geral da lógica fuzzy citamos o livro de Klir \& Yuan (1995), como internacional, e o livro de Barros \& Bassanezi (2006), como nacional.

\section{MATERIAL E MÉTODOS}

\subsection{Objetivos}

O objetivo geral deste trabalho foi apresentar um modelo, baseado em indicadores e Lógica Fuzzy, para quantificar conceitos subjetivos e relevantes à sustentabilidade de Floresta Ombrofila Mista.

Como objetivos específicos têm-se o estabelecimento de indicadores para a sustentabilidade de florestas de Araucaria Angustifolia; alimentar os indicadores com dados de Inventários Florestais já realizados; apresentar resultados para a Sustentabilidade da Floresta através de indicadores calculados por meio de Lógica Fuzzy. 


\section{2. Área de estudo}

A área de estudo está contida na Floresta Nacional (FLONA) de Irati, cuja manutenção atual é feita pelo Instituto Chico Mendes (ICMbio). A FLONA de Irati (Figura 1) localiza-se nos municípios de Irati e Fernandes Pinheiro, confrontando com Imbituva e Teixeira Soares, no Estado do Paraná; entre as coordenadas $\mathrm{E}=538.217,9 \mathrm{~m}$ a $\mathrm{E}=549.784,5 \mathrm{~m}$ e $\mathrm{N}=7.188 .444,2 \mathrm{~m}$ a $\mathrm{N}=7.201 .876,4$ do sistema UTM, pertencente ao fuso 22 (longitudes: $54^{\circ}$ e $48^{\circ}$ ), cujo meridiano central é 51, a uma altitude média de 885 metros (Figueiredo et al., 2006).

Os dados aqui apresentados foram coletados a partir de trabalhos anteriormente executados no fragmento de Floresta Ombrofila Mista. Nesse fragmento, em 2002 foram instaladas 25 parcelas permanentes, as quais estão subdivididas em blocos de 1 hectare, com quatro parcelas de 2.500 metros quadrados, contendo cada uma dessas parcelas cinco faixas. Nesta pesquisa utilizaram-se os dados do bloco 1 para alimentar os indicadores (Figura 2).

O clima da região é do tipo $\mathrm{Cfb}$, segundo a classificação de Köppen, isto é, subtropical úmido sem estação seca. As temperaturas médias dos meses mais quentes são inferiores a $22^{\circ} \mathrm{Ce}$, nos meses mais frios, são superiores a $10^{\circ} \mathrm{C}$; a temperatura média anual, por sua vez, é próxima de $17,2^{\circ} \mathrm{C}$, e constata-se a ocorrência de mais de cinco geadas noturnas por ano. A média anual de precipitação é de $1.442 \mathrm{~mm}$.

A FLONA de Irati é composta de Floresta Ombrofila Mista (FOM), caracterizada pela presença do pinheiro (Araucaria angustifólia), que apresenta valores de dominância, densidade e frequência bastante elevados em relação às demais espécies (Pottker, 2012).

Nos inventários realizados, constatou-se a ocorrência de 128 espécies arbóreas e arborescentes, pertencentes a 83 gêneros e 43 famílias botânicas (Galvão et al., 1989).

A área não está submetida a qualquer tipo de manejo desde que foi transformada em unidade de conservação, em 1942. Tal característica a equipara a uma floresta nativa inalterada, dotada de três estratos vegetativos.

\subsection{Inventário florestal}

O inventário florestal contínuo da Floresta Nacional de Irati (FLONA) é realizado pelo Laboratório de Manejo Florestal da Universidade do Centro-Oeste (UNICENTRO). O inventário teve início em 2002, quando foram definidos os limites das 25 parcelas permanentes e realizada a primeira medição. Em 2005 ocorreu a primeira remedição dos dados, seguida pela remedição de 2008 e. mais recentemente, pela de 2011.

Conforme exposto na Figura 2, as parcelas permanentes estabelecidas cobrem uma área de 25 hectares contíguos. A operacionalização do inventário forçou a subdivisão da amostra em 25 blocos de 1 hectare (100 m x $100 \mathrm{~m}$ ), cada bloco é formado por quatro parcelas de $2.500 \mathrm{~m}^{2}$ (50 m x $50 \mathrm{~m}$ ) e, finalmente, para cada uma dessas parcelas, cinco faixas são alocadas $(50 \mathrm{~m} \times 10 \mathrm{~m})$ (Figura 3). A regra para inclusão de uma árvore no banco de dados é possuir CAP igual ou superior a $31,4 \mathrm{~cm}$ (10 cm de DAP) (Pottker, 2012).

Dentre as características medidas nas parcelas permanentes ao longo de 9 anos estão a circunferência à altura do peito (CAP), as coordenadas das árvores

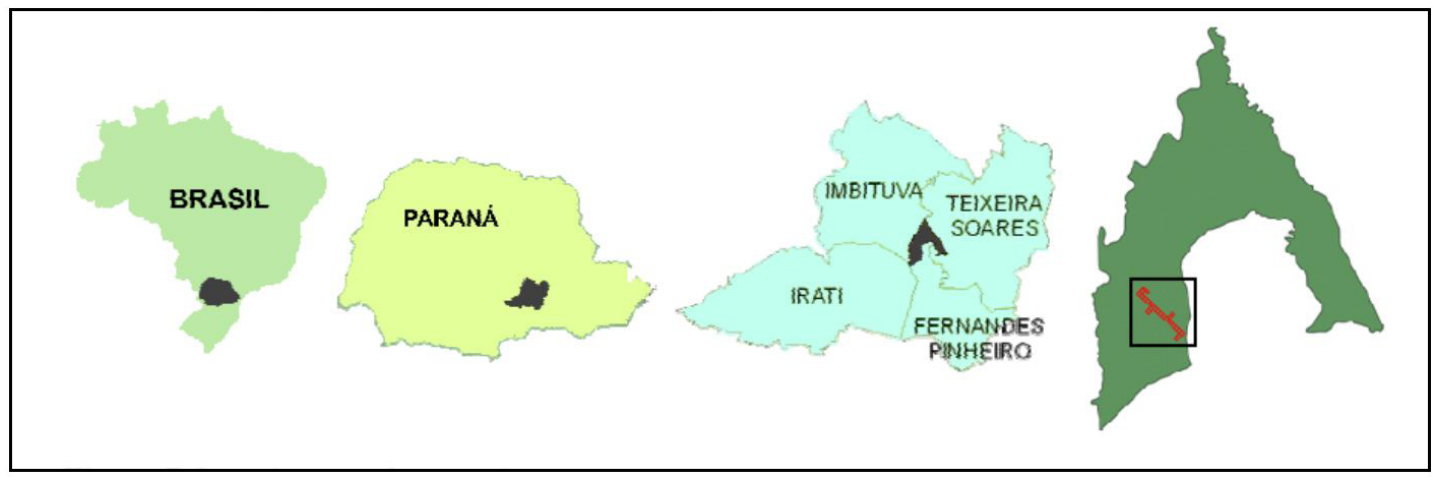

Figura 1. Localização da FLONA. Fonte: Pottker (2012, p. 46).

Figure 1. Location of FLONA. Source: Pottker (2012, p. 46). 


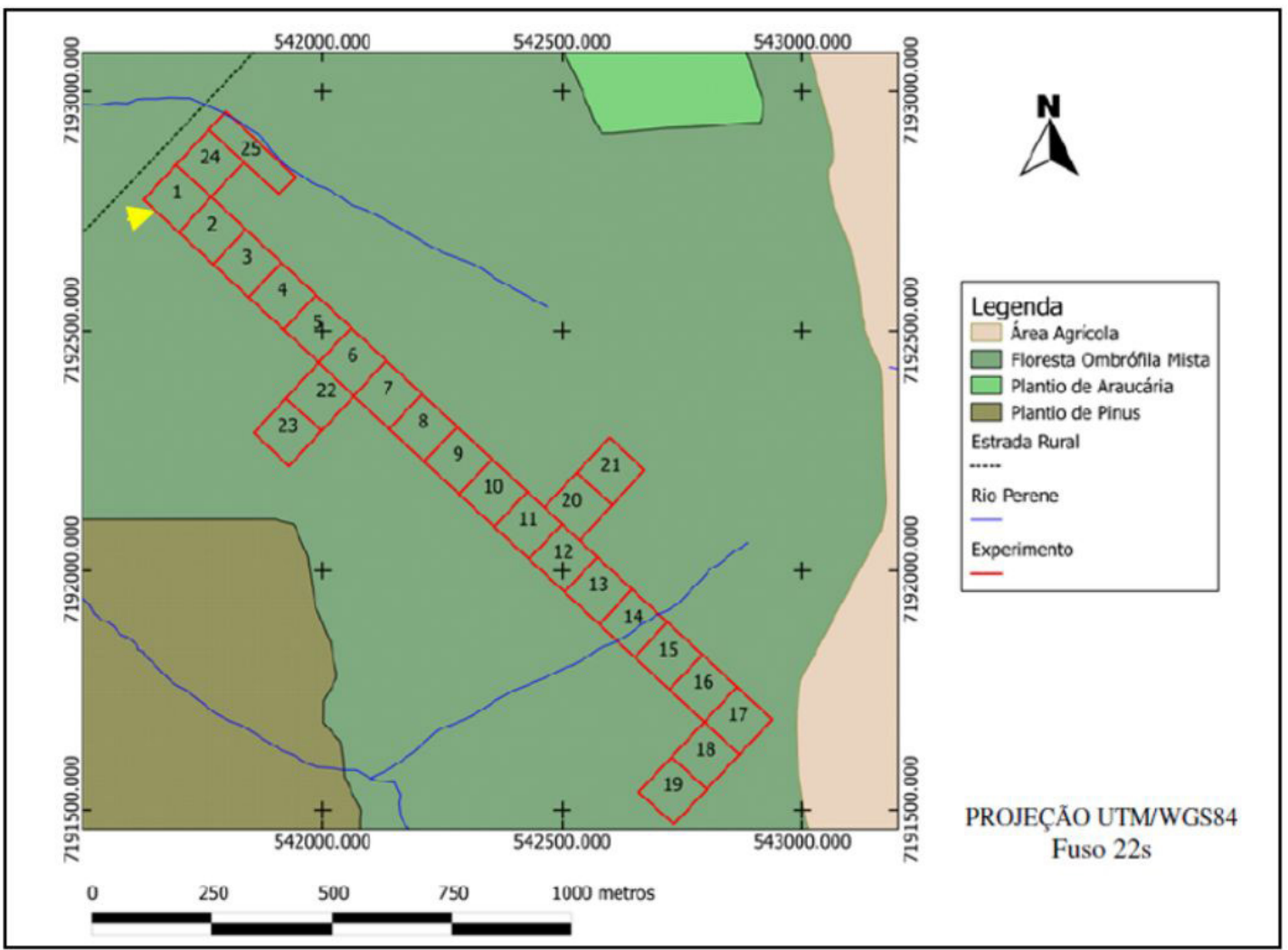

Figura 2. Localização dos blocos permanentes na FLONA de Irati, PR, com destaque do bloco 1. Fonte: adaptado de Pottker (2012, p. 46).

Figure 2. Location of permanent blocks on FLONA of Irati-PR with emphasis on block 1. Source: adapted from Pottker (2012, p. 46).

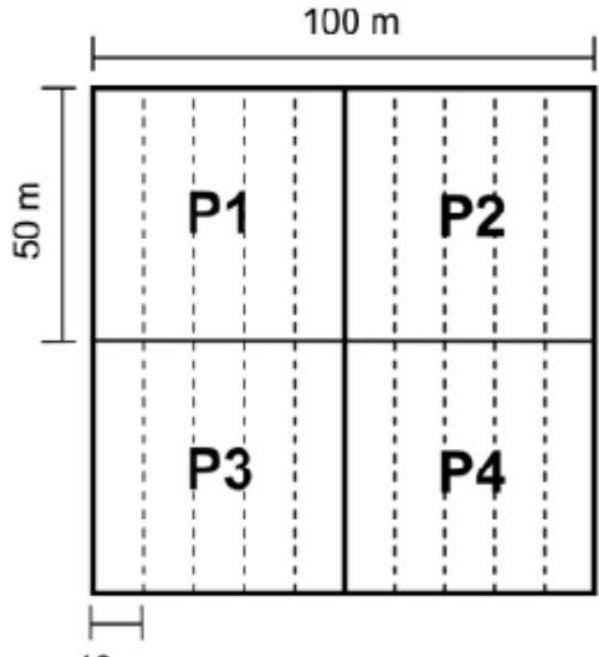

$10 \mathrm{~m}$

Figura 3. Esquema de um bloco contendo quatro parcelas com quatro faixas. Fonte: Rode (2008).

Figure 3. Schema of a block containing four parcels with four tracks. Source: Rode (2008). e uma série de classificações relativas à qualidade do fuste, à copa, ao estrato e à condição fitossanitária das árvores, dentre outras.

\subsection{Sistema de Inferência Fuzzy - SIF}

A classificação obtida pela lógica fuzzy se aproxima mais da forma do pensamento e da linguagem natural humana, dispondo de ferramentas mais apropriadas para a descrição dos critérios de decisão, sugerindo a possibilidade de obtenção de menores erros num processo classificatório (Ortega, 2001).

São estabelecidos indicadores para avaliação da sustentabidade do fragmento da floresta em questão, cujos parâmetros são classificados em conjuntos fuzzy. A modelagem nebulosa ou Fuzzy considera que existe uma zona de transição entre as classes, permitindo uma melhoria na representação dos fenômenos, constituindo assim um avanço na modelagem e nos processos de 
tomada de decisão (Maranhão, 2005). Os conjuntos fuzzy são definidos pelas funções denominadas "função de pertinência", que caracterizam o comportamento da variável representada. Para a construção do modelo previsto serão adotadas funções lineares de pertinência, por serem consideradas simples em estrutura e eficientes na produtividade (Ortega, 2001).

A combinação dos elementos através do Sistema de Inferência Fuzzy se dá por meio da construção da base de regras. A base de regras é o raciocínio fuzzy composto pela análise subjetiva das possíveis combinações entre as classes dos elementos que compõem o parâmetro calculado de acordo com o formato básico (IF \{antecedentes\} e THEN \{consequentes\}) apresentado pela Equação 1 (Ortega, 2001).

$$
\text { IF } x_{1} \text { is } A_{1} A N D x_{2} \text { is } A_{2} \text { THEN } y \text { is } B
$$

O modelo do Sistema de Inferência Fuzzy (SIF) adotado para a base de regras foi o Mamdani, cuja saída é constituída pela superposição de cada um dos consequentes. Esse procedimento se constitui na intersecção ou união dos conjuntos dos parâmetros envolvidos (Ortega, 2001).

O principal critério adotado na construção da base de regras é o conhecimento empírico que se tem a respeito do comportamento de cada elemento considerado (Mathworks, 2000). Tal conhecimento pode ser obtido com entrevista com especialistas como por meio de bibliografias específicas, conforme é o caso desta pesquisa.

O processamento pelo Sistema de Inferência Fuzzy resultará, com o parâmetro avaliado, num valor defuzzificado, ou seja, um valor dentro do intervalo $[0,1]$ no eixo das abscissas, que o classificará ao conjunto fuzzy cujo valor de pertinência seja maior no eixo das ordenadas (Maranhão, 2005).

No Sistema de Inferência Fuzzy consideram-se as operações de intersecção e de união entre os dados envolvidos, visando alcançar maior expressividade no resultado que se deseja encontrar, sendo que a intersecção e união dos conjuntos fuzzy equivalem, respectivamente, ao "AND" e ao "OR" Booleano. O resultado proveniente do sistema, SIF, consta de um valor determinístico (defuzzyficado), quantificador, que favorece as análises por comparações, e, também, de um valor semântico, que é mais adequado para os processos de tomada de decisão (Ortega, 2001).
No desenvolvimento de modelos processados pelo SIF é altamente significativa a intervenção do usuário na construção das bases de regras. Isso torna o modelo flexível às necessidades e particularidades de cada situação (Lazzarotto, 2005).

O modelo inicial para o cálculo do Indicador da Sustentabilidade da Floresta é proposto conforme indicado na Figura 4. O levantamento de dados para esta pesquisa, bem como os valores limites para os conjuntos fuzzy se encontram na Tabela 1.

\subsection{Construção dos indicadores}

O indicador INA - Incremento do Número de Árvores foi construído pela interseção dos dados de Ingresso e Mortalidade de árvores previamente classificados em conjuntos fuzzy relativos ao número de árvores ingressas ou mortas, adotando-se os seguintes critérios:

Ingresso e Mortalidade (Figura 5):

Fenômenos analisados (ingresso e mortalidade): [0 a 54];

- BAIXO: $(0,0,27)$;

- MÉDIO: $(0,27,54)$;

- ALTO: $(27,54,54,54 \ldots)$.

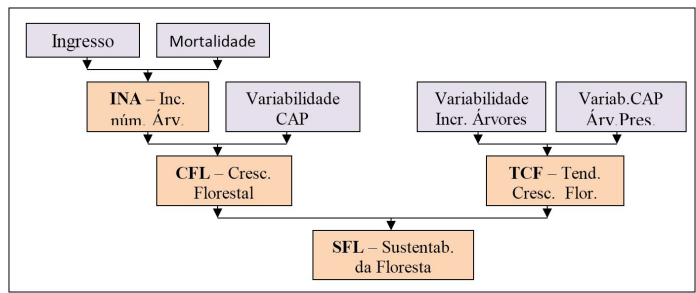

Figura 4. Modelo de indicadores para avaliação da Sustentabilidade da Floresta.

Figure 4. Indicators model to evaluate the sustainability of forest.

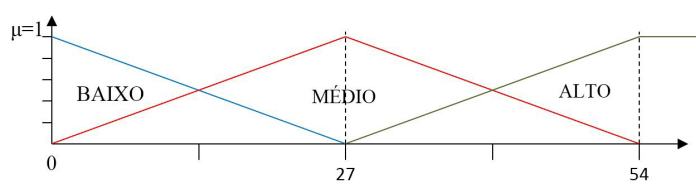

Figura 5. Classificação em conjuntos fuzzy para o ingresso e a mortalidade de árvores.

Figure 5. Classification fuzzy sets to: admission and mortality of trees. 
Incremento no Número de Árvores - INA (Figura 6):

Fenômenos analisados concomitantemente (Ingresso e Mortalidade): [-54, 54];

- RB - REDUÇÃO BAIXA: (-54, -54, -5);

- RM - REDUÇÃO MÉDIA: (-54, -5, -5);

- EQUIL - EQUILÍBRIO: (-5, -5, 5, 5);

- AB - AUMENTO BAIXO: $(5,5,54)$;

- AA - AUMENTO ALTO: $(5,54,54,54 \ldots)$.

As bases de regras são construídas com base na lógica da Equação 1. Para a base de regras visando a obtenção do indicador INA - Incremento no Número de Árvores, considera-se a interseção dos conjuntos Ingresso e Mortalidade, combinando-se suas classes conforme Quadro 1.

O indicador CFL - Crescimento Florestal foi construído pela interseção dos dados do indicador Incremento Número de Árvores (INA) e da Variabilidade de CAP, segundo a classificação e conjuntos fuzzy descritos a seguir (Figura 7).

Indicador INA, já descrito anteriormente e representado na Figura 6;

Variabilidade de CAP (somatório total do CAP em cada medição).

O máximo valor observado para (CAP): [0 a 1492];

- BAIXO: $(0,0,746)$;

- MÉDIO: (0, 746, 1.492);

- ALTO: (746, 1.492, 1.492, 1.492...).

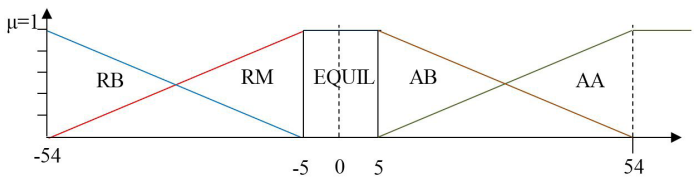

Figura 6. Classificação em conjuntos fuzzy para o indicador INA.

Figure 6. Classification fuzzy sets for the INA indicator.

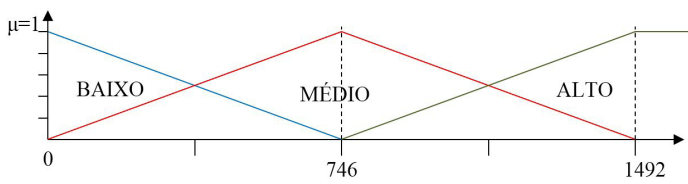

Figura 7. Classificação fuzzy para os dados referentes ao CAP.

Figure 7. Fuzzy classification to data relating to CAP.
CFL - Crescimento florestal $[0,1]$ :

- MUITO BAIXO: $(0,0,0,25)$;

- BAIXO: $(0,0,25,0,5)$;

- MÉDIO: $(0,25,0,5,0,75)$;

- ALTO: $(0,5,1,1)$;

- MUITO ALTO: $(0,75,1,1)$.

Base de regras para obtenção do indicador CFL - Crescimento Florestal considera a interseção dos conjuntos do indicador Incremento Número de Árvores (INA) e Variabilidade de CAP, combinando suas classes conforme Quadro 2.

$\mathrm{O}$ indicador TCF - Tendência de Crescimento Florestal foi construído pela interseção dos dados de Variabilidade no Incremento do número de Árvores e Variabilidade do CAP entre Árvores Presentes em pelo menos duas medições consecutivas, ou seja, sem considerar ingressos e mortes computados no somatório total de cada medição isolada. A classificação e conjuntos fuzzy desses parâmetros são descritos a seguir.

Variabilidade no Incremento do número de Árvores $[-16,16]$ :

- VNMB - VARIAÇÃO NEGATIVA MUITO BAIXA: $(-16,-16,0)$

- VNB - VARIAÇÃO NEGATIVABAIXA: $(-16,0,16)$;

- VPB - VARIAÇÃO POSITIVA BAIXA: $(0,0,16)$;

• VPA-VARIAÇÃO POSITIVAALTA: $(0,16,16,16 \ldots)$;

Quadro 1. Base de regras para o indicador INA.

Chart 1. Rules base to the INA indicator.

\begin{tabular}{ccccc} 
& & \multicolumn{3}{c}{ MORTALIDADE } \\
& & DE ÁRVORES \\
\cline { 3 - 5 } & & ALTA & MÉDIA & BAIXA \\
\hline INGRESSO & ALTO & EQUIL & AB & AA \\
DE & MÉDIO & RB & EQUIL & AB \\
ÁRVORES & BAIXO & RA & RB & EQUIL \\
\hline
\end{tabular}

Quadro 2. Base de regras para o indicador CFL.

Chart 2. Rules base for the CFL indicator.

\begin{tabular}{cccccccc} 
& & \multicolumn{5}{c}{ INCREMENTO NÚM. } \\
& & & ÁRVORES \\
\cline { 3 - 8 } & RA & RB & EQUIL & AB & AA \\
\hline \multirow{2}{*}{$\begin{array}{c}\text { VARIAÇÃO } \\
\text { CAP }\end{array}$} & ALTA & MB & MB & B & B & M \\
& MEIXIA & B & B & M & M & A \\
& BAIXA & A & B & M & M & MA \\
\hline
\end{tabular}


Dados de Variabilidade no Incremento do CAP em árvores presentes em pelo menos duas medições consecutivas $[0,1492]$ :

- BAIXA: $(0,0,746)$;

- MÉDIA: (0, 746, 1.492);

- ALTA: $(0,1.492,1.492,1.492 \ldots)$.

TCF - Tendência do Crescimento Florestal $[0,1]$ (Figura 8):

- DECRESCENTE: $(0,0,0,25)$;

- MANTÉM-SE: $(0,0,25,0,5)$;

- CB - CRESCIMENTO BAIXO: $(0,25,0,5,0,75)$;

- CM - CRESCIMENTO MÉDIO: $(0,5,0,75,1)$;

- CA - CRESCIMENTO ALTO: $(0,75,1,1)$.

Base de regras para obtenção do indicador TCF - Tendência de Crescimento Florestal considera a interseção dos conjuntos relativos aos parâmetros: Variabilidade no Incremento do número de Árvores, e Variabilidade no CAP de Árvores Presentes em pelo menos duas medições consecutivas, combinando suas classes conforme Quadro 3.

O indicador da SF - Sustentabilidade da Floresta foi construído pela interseção dos indicadores CFL - Crescimento Florestal e TCF - Tendência de Crescimento Florestal. A classificação e conjuntos fuzzy desses parâmetros já foram descritos anteriormente e representados pelas Figuras 9 e 10, respectivamente.

Sustentabilidade da Floresta apresenta as seguintes características $[0,1]$ (Figura 10):

- NS - NÃO SUSTENTÁVEL - situação de decadência da floresta $(0,0,0,25)$;

- SB - SUSTENTABILIDADE BAIXA - situação precária para a sustentabilidade da floresta com eventuais baixas $(0,0,25,0,5)$;
• EQUIL - EQUILÍBRIO - situação estável para a sustentabilidade da floresta $(0,25,0,5,0,75)$;

- SM - SUSTENTABILIDADE MÉDIA - boa situação para a sustentabilidade da floresta, havendo inclusive algum incremento florestal $(0,5,0,75,1)$;

- S - SUSTENTÁVEL - excelentes condições para a sustentabilidade da floresta, havendo grandes avanços de incremento florestal $(0,75,1,1)$.

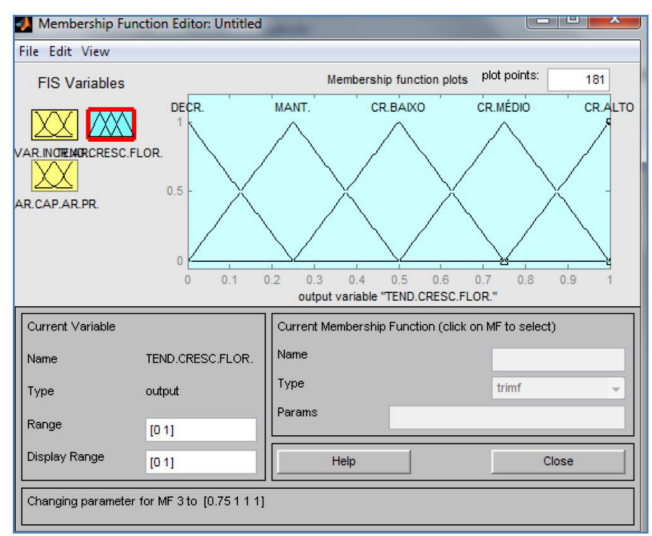

Figura 8. Classificação fuzzy dos parâmetros para o cálculo do indicador TCF.

Figure 8. Fuzzy classification of parameters for calculate of TCF.

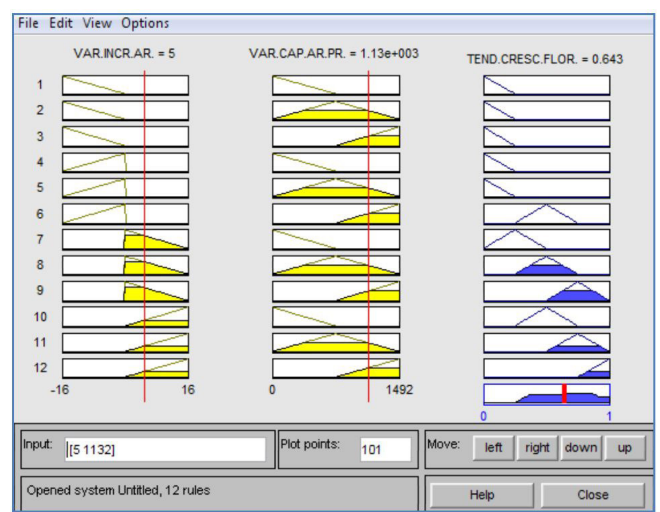

Figura 9. Resultado do sistema de inferência fuzzy para o indicador TCF.

Figure 9. Result of the fuzzy inference system for TCF indicator.

Quadro 3. Base de regras para o indicador TCF.

Chart 3. Rules base for TCF indicator.

\begin{tabular}{|cccccc|} 
& & \multicolumn{4}{c|}{ VARIAÇÃO DO INCR. NÚM. ÁRVORES } \\
\cline { 3 - 6 } & & VNMB & VNB & VPB & VPA \\
\hline \multirow{2}{*}{ VARIAÇÃO CAP DE } & BAIXA & DECR & DECR & MANT & CB \\
ÁRV. PRESENT. 2 M. & MÉDIA & DECR & DECR & CB & CM \\
& ALTA & DECR & CB & CM & CA \\
\hline
\end{tabular}


A base de regras para obtenção do indicador SF - Sustentabilidade da Floresta considera a interseção dos conjuntos relativos aos indicadores: CFL - Crescimento Florestal e TCF - Tendência de Crescimento Florestal, conforme Quadro 4.

\section{RESULTADOS E DISCUSSÃO}

Os dados de entrada para o cálculo dos Indicadores propostos no Modelo foram extraídos do Banco de Dados relativos aos inventários florestais realizados em 2002, 2005, 2008 e 2011 no fragmento da Floresta Nacional de Irati (FLONA). O Bloco 1 foi a área considerada para o levantamento de dados para esta pesquisa, conforme Tabela 1.

A seguir apresentam-se os resultados do processamento dos indicadores descritos anteriormente.

Resultado do indicador INA - Incremento no Número de Árvores, para os seguintes valores de entrada:

- Valor médio de Ingresso: 35,67 ;

- Valor médio de Mortalidade: 30,67.

Resultando no valor defuzzificado igual a 6,44 , conforme Figura 11.

O valor defuzzificado de 6,44 apresenta um pertencimento maior para a classe $A B$ (próximo de $\mu=1$, ou seja, maior que $90 \%$ ), do que para a classe AA (próximo de $\mu=0$ ), portanto, classifica o indicador INA na classe $A B$ - Aumento Baixo, ou seja, houve pequeno aumento no número de árvores, conforme mostra a Figura 12, concordando com a realidade dos dados.

Resultado do CFL - Crescimento Florestal, para os seguintes valores de entrada:

- Considerando o resultado obtido para o indicador INA: 6,44;

Quadro 4. Base de regras para o indicador SF.

Chart 4. Base rules for SF indicator.

\begin{tabular}{|c|c|c|c|c|c|c|}
\hline & \multicolumn{5}{|c|}{$\begin{array}{l}\text { CRESCIMENTO } \\
\text { FLORESTAL }\end{array}$} \\
\hline & & MB & B & M & A & MA \\
\hline \multirow{5}{*}{$\begin{array}{l}\text { TENDÊNCIA } \\
\text { DE CRESC. } \\
\text { FLORESTAL }\end{array}$} & DECR & NS & NS & NS & NS & NS \\
\hline & MANT & NS & NS & SB & EQ & EQ \\
\hline & CB & NS & SB & EQ & SM & SM \\
\hline & CM & NS & SB & EQ & SM & S \\
\hline & $\mathrm{CA}$ & SB & EQ & SM & S & S \\
\hline
\end{tabular}

- A Variabilidade do CAP (a cada 3 anos): 512.

Esses dados apresentaram resultado defuzzificado de 0,463 , conforme Figura 13 .

O valor defuzzificado de 0,463 para CFL apresenta um pertencimento maior para a classe MÉDIO, cujo intervalo considerado foi de 0,25 a 0,75 , classificando-o, portanto, nessa classe.

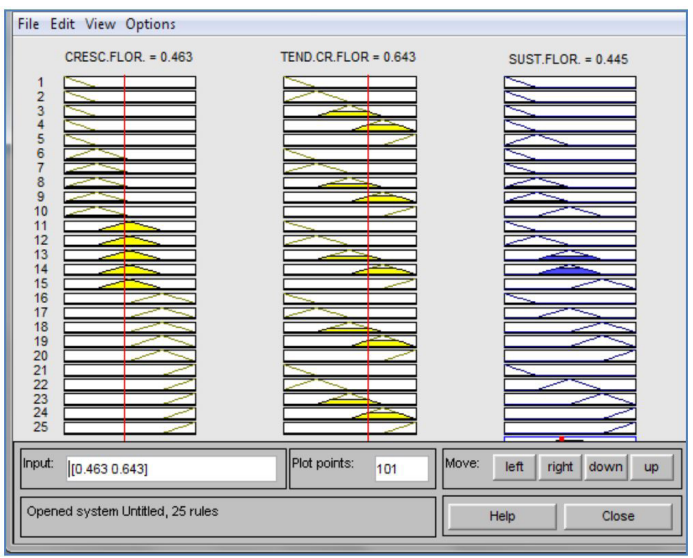

Figura 10. Resultado do sistema de inferência fuzzy para o indicador SF.

Figure 10. Result of the fuzzy inference system for the $\mathrm{SF}$ indicator.

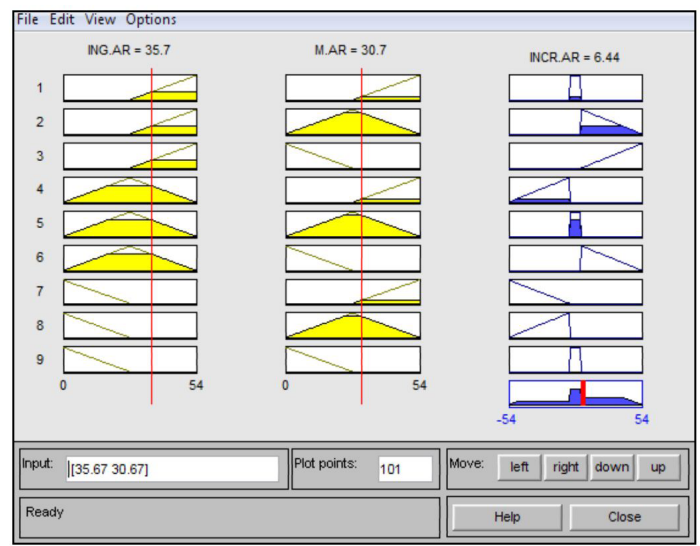

Figura 11. Resultado do sistema de inferência fuzzy para o indicador INA.

Figure 11. Result of the fuzzy inference system to the INA indicator.

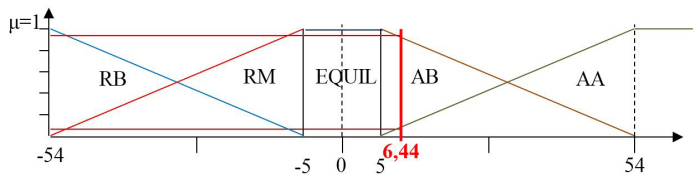

Figura 12. Esquema do resultado para o indicador INA. Figure 12. Scheme of the result for the INA indicator. 
Tabela 1. Dados do Bloco 1 - Inventários florestais na FLONA Irati, PR.

Table 1. Data Block 1 - Forest inventories in FLONA Irati-PR.

\begin{tabular}{|c|c|c|c|c|c|c|c|c|c|}
\hline \multicolumn{3}{|c|}{ BLOCO 01} & PARÂMETRO & 2002 & 2005 & 2008 & 2011 & $\begin{array}{c}\text { TOTAL } \\
2002-2011\end{array}$ & Med/3ano \\
\hline \multirow{7}{*}{\multicolumn{2}{|c|}{ 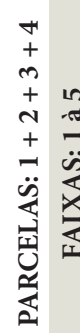 }} & \multirow{4}{*}{$\frac{3}{0}$} & Ingresso & - & 54 & 27 & 26 & $107(683)$ & 35,67 \\
\hline & & & Mortalidade & - & 38 & 34 & 20 & $92(484)$ & 30,67 \\
\hline & & & N. Árvores & 576 & 592 & 585 & 591 & & \\
\hline & & & Var - N. Árvores & - & +16 & -7 & +6 & +15 & +5 \\
\hline & & \multirow{3}{*}{ ใ্ঠ } & CAP & 40847,7 & 42172,6 & 41888,6 & 42386,55 & & \\
\hline & & & Var. - CAP & - & $+1324,9$ & $-284,0$ & $+497,95$ & $+1538,85$ & $+512,95$ \\
\hline & & & $\begin{array}{l}\text { Var.- CAP (arv.exist.em } \\
\text { duas med. consecutivas) }\end{array}$ & $(40847,7)$ & 1492 & 1012,2 & 890,4 & 3394,5 & $+1131,5$ \\
\hline
\end{tabular}

Resultado do processamento do indicador TCF - Tendência do Crescimento Florestal, para os seguintes valores de entrada:

- Valor médio (3 anos) da Variabilidade no Incremento do número de Árvores $=5$;

- Valor médio (3 anos) da Variabilidade do CAP entre Árvores Presentes em pelo menos duas medições consecutivas $=1.131,5$.

Resultando no valor defuzzificado igual a 0,643 , conforme Figura 9.

O valor defuzzificado de 0,643 para TCF apresenta um pertencimento maior para a classe CRESCIMENTO MÉDIO, classificando-o, portanto, nesta classe.

De forma similar aos demais indicadores, o resultado do processamento do indicador SF - Sustentabilidade da Floresta, para os seguintes valores de entrada, resultou no valor defuzzificado igual a 0,445 , conforme Figura 10.

- Valor do indicador CFL: 0,463;

- Valor do indicador TCF: 0,643.

A representação dos conjuntos fuzzy referentes ao indicador de Sustentabilidade da Floresta com o resultado obtido pela aplicação do modelo proposto (Figura 4) e processado pelo sistema de inferência fuzzy apresenta-se na Figura 14. Nessa figura observa-se a localização do valor defuzzificado, 0,445 , no eixo das abscissas, e as respectivas pertinências, no eixo das ordenadas, aos conjuntos EQ (EQUILÍBRIO), com o maior valor 0,78, e SB (SUSTENTABILIDADE BAIXA), com o menor valor 0,23 . Portanto, o resultado obtido classifica o indicador de Sustentabilidade da Floresta

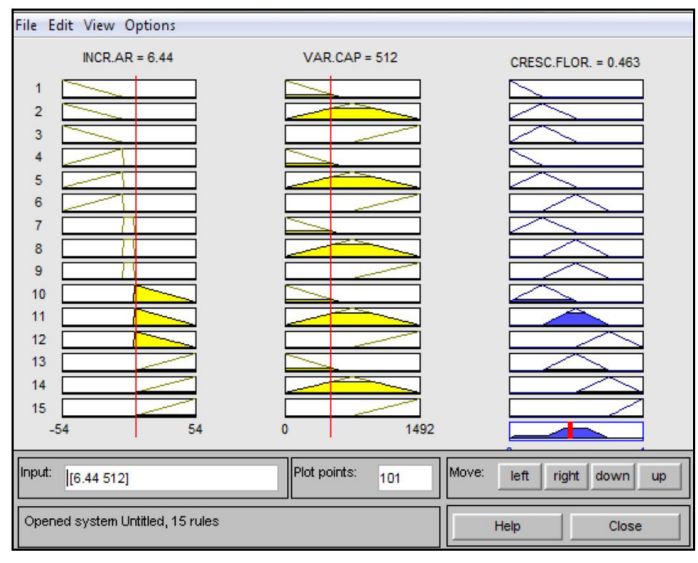

Figura 13. Resultado do sistema de inferência fuzzy para o indicador CFL.

Figure 13. Result of the fuzzy inference system for the CFL indicator.

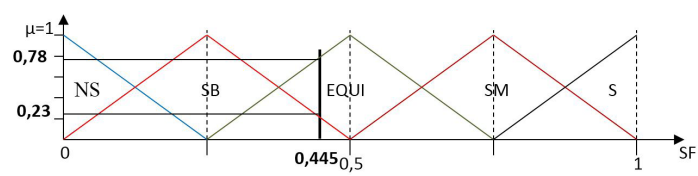

Figura 14. Classificação fuzzy e resultado para o indicador SF.

Figure 14. Fuzzy classification and result for the SF indicator.

para o fragmento analisado como estando em situação de EQUILÍBRIO.

Esse resultado mostra que o Modelo adotado está coerente, pois tratando-se de um fragmento de floresta natural preservado já se esperava por tal resultado. Embora a análise aqui elaborada tenha sido efetuada apenas em uma pequena parcela de toda a área florestal, o resultado deve alertar a consciência de que o grau de sustentabilidade revelado não é significativamente 
forte para justificar atitudes de acomodação. Faz-se necessário monitoramento contínuo para garantir a manutenção da floresta. Recomenda-se a continuidade dessas pesquisas abrangendo a totalidade dos 25 ha e ainda agregando-se ao Modelo parâmetros relativos a Fuste, Estrato, Estado Fitossanitário e Copa na construção de um indicador relativo à saúde da floresta.

\section{CONCLUSÃO}

O indicador INA - Incremento no Número de Árvores ficou classificado como AUMENTO BAIXO, com valor defuzzificado igual a 6,44 - o intervalo desse conjunto é de 5 à 54 (Figura 6). Esse resultado concorda com os dados de entrada, nos quais a média de incremento no número de árvores foi igual a cinco (Tabela 1).

O indicador CFL - Crescimento Florestal, com valor defuzzificado igual a 0,463, obteve classificação no conjunto MÉDIO, cujo intervalo é de 0,25 à 0,75.

$\mathrm{O}$ indicador TCF - Tendência do Crescimento Florestal foi classificado como CRESCIMENTO MÉDIO pelo valor defuzzificado de 0,643 , portanto dentro do conjunto de intervalo entre 0,5 e 1 (Figura 8). Tanto o resultado do indicador CFL como o do TCF acordam com os números reais levantados nos inventários, conforme se pode constatar pela variação do CAP, que tem valor positivo de 512,95 mostrado na Tabela 1 .

E, finalmente, o indicador SFL - Sustentabilidade da Floresta obteve valor defuzzificado de 0,445 , portanto, dentro do conjunto EQUILÍBRIO, cujo intervalo é de 0,25 a 0,75 .

Com os resultados obtidos, concluímos que o fragmento da floresta analisada está com um valor de pertencimento de $78 \%(\mu=0,78)$ à classe EQUILÍBRIO. Esse resultado está coerente com a realidade da floresta que, por pertencer a uma área de proteção, segue livre das atividades antrópicas. Um resultado mais favorável no futuro será possível se houver um programa de incremento da floresta, visando sua expansão, pois o estado de equilíbrio em porções pequenas, conforme é o caso, pode vir a sofrer degradação com a expansão urbana ao seu redor. Além da preservação, recomenda-se o fomento da floresta.

O presente trabalho cumpriu seu objetivo de propor um modelo para avaliar a sustentabilidade da floresta e seu resultado retratou a realidade.
O modelo pode facilmente agregar qualquer quantidade de parâmetros, de acordo com o que se dispõe ou se deseja avaliar. Certamente, a flexibilidade do modelo na combinação de parâmetros, bem como na atribuição de pesos na construção da base de regras necessárias ao sistema de inferência fuzzy é outra importante vantagem, uma vez que possibilita evidenciar aspectos de interesses específicos.

\section{AGRADECIMENTOS}

Esta pesquisa só foi possível devido ao acesso aos dados dos inventários florestais da FLONA de Irati, $\mathrm{PR}$, realizado principalmente por pesquisadores da Unicentro e sob supervisão do professor dr. Afonso Figueiredo Filhos. A eles registramos aqui nossos sinceros agradecimentos.

\section{STATUS DA SUBMISSÃO}

Recebido: 6 set., 2012

Aceito: 23 fev., 2017

\section{AUTOR(ES) PARA CORRESPONDÊNCIA}

\section{Deise Regina Lazzarotto}

Departamento de Engenharia Ambiental, Universidade Federal da Fronteira Sul - UFFS, Rodovia SC 484, Km 2, Fronteira Sul, CEP 89815-899, Chapecó, SC, Brasil e-mail: deiselazza@gmail.com

\section{REFERÊNCIAS}

Akdemir HG, Tiryaki F. Using fuzzy linear regression to estimate relationship between forest fires and meteorological conditions. Applications and Applies Mathematics 2013; 8(2), 673-683.

Amendola M, Souza AL, Barros LC. Manual do uso da teoria dos conjuntos Fuzzy no MATLAB 6.5. Campinas: FEAGRI \& IMECC; UNICAMP; 2005.

Barros LC, Bassanezi RC. Tópicos de Lógica Fuzzy e Biomatemática. Campinas: Universidade Estadual de Campinas; 2006. (Coleção IMECC. Textos Didáticos; vol. 5).

Bolfe EL, Pereira RS, Madruga PRA, Nunes GM. Gerenciamento de unidades de produção florestal através de técnicas de geoprocessamento em lógica nebulosa (Logic Fuzzy). In: Anais do X SBSR; 2001; Foz do Iguaçu. Foz do Iguaçu: INPE; 2001. p. 921-923. 
Chang M. Seqüestro florestal de carbono no Brasil: dimensões políticas, socioeconômicas e ecológicas. São Paulo: Annablume; IEB; 2004. 278 p.

Chen B, Hudson C, Grawford A, Kim M, Che D. Adaptivelybranching Fuzzy Greedy K-mean Decision Forest (FGKDF) model for protein local tertiary structure prediction. American Journal of Bioinformatics and Computational Biology 2014; 2(1), 1-19. http://dx.doi.org/10.7726/ ajbcb.2014.100.

Figueiredo A Fo, Dias AN, Wazlawick LF. Inventário das florestas naturais na Floresta Nacional de Irati, Estado do Paraná. Irati: Universidade Estadual do Centro-Oeste; 2006.

Galvão F, Kuniyoshi YS, Roderjan CV. Levantamento fitossociológico das principais associações arbóreas da Floresta Nacional de Irati, PR. Revista Floresta 1989; 19(1): 30-49.

Klir GJ, Yuan B. Fuzzy sets and fuzzy logic: theory and applications. New Jersey: Prentice Hall; 1995. 574 p.

Lazzarotto DR. Avaliação da Qualidade de Base Cartográfica por meio de Indicadores e Sistema de Inferência Fuzzy [tese]. Curitiba: Departamento de Geomática, Universidade Federal do Paraná; 2005.

Maranhão MRA. Modelo de seleção de áreas para atualização do mapeamento sistemático baseado em lógica nebulosa [dissertação]. Rio de Janeiro: Universidade Estadual do Rio de Janeiro; 2005.

Mathworks. MatLab 5.3. User's guide. Fuzzy Lógic Toolbox. Massachusetts: Mathworks; 2000.

Mendoza GA, Prabhu R. Fuzzy methods for assessing criteria and indicators of sustainable Forest management. Ecological Indicators 2003; 3(4): 227-236. http://dx.doi. org/10.1016/j.ecolind.2003.08.001.

Ortega NRS. Aplicação da Teoria de Conjuntos Fuzzy a Problemas da Biomedicina [tese]. São Paulo: Instituto de Física da Universidade de São Paulo; 2001.

Pottker GS. Distribuição Espacial de Espécies da Floresta Ombrófila Mista na FLONA de Irati, Paraná [dissertação]. Irati: Universidade Estadual do Centro Oeste; 2012.

Rode R. Avaliação florística e estrutural de uma Floresta Ombrófila Mista e de uma vegetação arbórea estabelecida sob um povoamento de Araucaria angustifolia de 60 anos [dissertação]. Curitiba: Universidade Federal do Paraná; 2008.

Silva IDB, Pontes ACF Jr. Elaboração de um fator de risco de incêndios florestais utilizando Lógica fuzzy. Biomatemática 2011; 21: 113-128. 